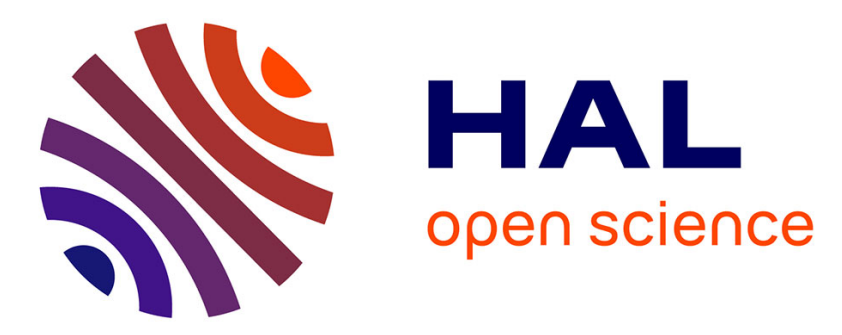

\title{
Modelling photophysical properties of metal-organic frameworks: a density functional theory based approach
}

\author{
Liam Wilbraham, François-Xavier Coudert, Ilaria Ciofini
}

\section{To cite this version:}

Liam Wilbraham, François-Xavier Coudert, Ilaria Ciofini. Modelling photophysical properties of metal-organic frameworks: a density functional theory based approach. Physical Chemistry Chemical Physics, 2016, 18 (36), pp.25176-25182. 10.1039/C6CP04056J . hal-02116956

\section{HAL Id: hal-02116956 https://hal.science/hal-02116956}

Submitted on 1 May 2019

HAL is a multi-disciplinary open access archive for the deposit and dissemination of scientific research documents, whether they are published or not. The documents may come from teaching and research institutions in France or abroad, or from public or private research centers.
L'archive ouverte pluridisciplinaire HAL, est destinée au dépôt et à la diffusion de documents scientifiques de niveau recherche, publiés ou non, émanant des établissements d'enseignement et de recherche français ou étrangers, des laboratoires publics ou privés. 


\title{
Modelling photophysical properties of metal-organic frameworks: A Density Functional Theory based approach
}

\author{
Liam Wilbraham, François-Xavier Coudert*, Ilaria Ciofini*
}

Chimie ParisTech, PSL Research University, CNRS, Institut de Recherche de Chimie Paris, 75005 Paris, France

\begin{abstract}
Design of optical properties within metal-organic frameworks (MOFs) is a subject of ever increasing attention in recent years with theoretical approaches poised to play a key role alongside experiment in both the understanding of fundamental mechanisms and the further development of high performance materials. We have developed and applied a simple and computationally affordable protocol rooted in density functional theory (DFT) and its time dependent counterpart (TD-DFT) to two isostructural MOFs based on a 4,4'-bis((3,5-dimethyl-1H-pyrazol-4-yl)methyl)-biphenyl ( $\left.\mathrm{H}_{2} \mathrm{DMPMB}\right)$ linker. These systems show a remarkable dependence of photoluminescence properties on the interchange of zinc and cadmium cations as building units. Our investigation was able to successfully rationalize the subtle change in the photoluminescence mechanism experimentally observed responsible for the large $(0.88 \mathrm{eV})$ red shift (from $335 \mathrm{~nm}$ to $441 \mathrm{~nm})$ observed when going from the cadmium to the zinc based structure. More generally, this computational protocol seems well adapted for the characterization and rationalization of the absorption and emission behaviour of such complex extended materials.
\end{abstract}

\section{Authors for correspondence}

fx.coudert@chimie-paristech.fr and ilaria.ciofini@,chimie-paristech.fr 


\section{Introduction}

Coordination polymers and the related metal-organic frameworks (MOFs) have been the recipients of tremendous scientific interest throughout the last decade ${ }^{1}$. These materials are constructed from inorganic building units - consisting of either metal cations or inorganic cluster arrangements which are connected via coordinating organic linker molecules to form three-dimensional frameworks. The structural versatility and multifunctional nature of these frameworks have inspired research into their potential application in areas such as gas storage ${ }^{2}$, catalysis ${ }^{3}$ and sensing ${ }^{4}$.

Although the structural properties of MOFs have been investigated extensively both from experimental and a theoretical ${ }^{5-7}$ points of view, the electronic properties of these materials have recently become an area of increasing interest - with particular attention paid to enhanced photoluminescence — with the idea of exploiting these properties in areas such as small molecule sensing, non-linear optics and biomedical applications ${ }^{8}$. There is a particular interest in photoresponsive frameworks, which respond to light absorption by changes in their structure, or stimuli-responsive photoluminescent frameworks, whose optical properties are modified by external physical or chemical stimulations ${ }^{9,10}$.

While luminescence from MOF structures is a frequently observed phenomenon ${ }^{11,12}$, little effort thus far has been invested to gain a complete understanding of these excited state processes. There are various potential mechanisms cited in the literature which can give rise to intrinsic luminescence effects in MOFs differentiated by the different types of involved. The main processes discussed are indeed categorized as ligand centred (LC) emission ${ }^{13}$, metal centred (MC) emission ${ }^{14}$, ligand to metal charge transfer $(\mathrm{LMCT})^{15}$, metal to ligand charge transfer $(\mathrm{MLCT})^{16}$ and interligand charge transfer $(\text { LLCT })^{17}$ transitions. In addition, guest induced emission has also been observed either via encapsulation of luminescent molecules within pores $^{18}$ or via exciplex formation ${ }^{19}$.

Given the very nature of metal-organic frameworks is that an organic linker molecule is coordinated to an inorganic building unit (often a transition metal cation), there can often be increased efficiency of intersystem crossing (ISC) due to spin orbit coupling ${ }^{20}$, allowing emission from spin forbidden states centred on ligand moieties to become more readily accessible. It is therefore not surprising that 
competing effects are often observed leading to multiple simultaneous emission types in $\mathrm{MOFs}^{13}$. However, identifying emission routes and discerning between competing processes is usually difficult from an experimental point of view. Given this array of possibilities, it could be useful to have tools at one's disposal which can be employed to distinguish between these mechanisms and their subtle, yet often profound consequences.

In this respect, gaining a more complete understanding of excited state behaviour in MOFs from a theoretical perspective could be a powerful tool, aiding in the rational design of high-performance luminescent materials. With this in mind, this paper attempts to improve upon the current understanding of MOF luminescence via the design of a suitable quantum computational protocol, rooted in density functional theory (DFT) and its time-dependent counterpart (TD-DFT), for the characterisation and prediction of MOF luminescence behaviour. This protocol seeks to adopt a dual approach - based on calculations utilising both periodic-boundary conditions and molecular clusters to assess and discern the role of the crystalline environment, from a geometrical point of view, on the computed luminescence properties. It is also of interest to have a unified computational protocol namely to have a consistent approach in terms of functional and basis to obtain both geometrical and photophysical features.

To this end, we have chosen an appropriate case study of a pair of MOF structures composed of a 4,4bis((3,5-dimethyl-1H-pyrazol-4-yl)methyl)biphenyl ( $\mathrm{H}_{2}$ DMPMB) ligand and interchangeable metal cations of zinc and cadmium, recently characterised experimentally by Tabacaru and co-workers ${ }^{22}$, hereafter labelled M[DMPMB] $(\mathrm{M}=\mathrm{Zn}, \mathrm{Cd})$. With an orthorhombic structure, these two frameworks crystallise in near-identical structures (in the Pccn space group), depicted in Figure 1 along with the protonated ligand structure. Each transition metal ion is coordinated by four ligand molecules in a distorted tetrahedral fashion. The nitrogen atoms of a pyrazole ring each bonding to a different $\mathrm{M}$ atom by their lone pair, each ligand thus bridging between two metal ions. The biphenyl groups of neighbouring ligands are oriented on perpendicular planes, giving rise to the possibility of T-shaped $\pi-\pi$ interactions. While other MOFs constructed using this linker were reported, the zinc and cadmium frameworks were specifically selected as they are isostructural - allowing one to directly 
probe the effects of changing the coordinating metal - and both exhibit interesting luminescence properties. Furthermore, the simple interchange of the coordinating metal from zinc to cadmium results in a $0.88 \mathrm{eV}$ (that is, from $441 \mathrm{~nm}$ to $335 \mathrm{~nm}$ ) blue shift and thus is an interesting and novel example of tuneable luminescence in metal-organic frameworks. Despite this, no explanation for this rather large change in emission wavelength was proposed in the literature.

The paper is structured as follows: first, a computational protocol is determined by screening the performance of various exchange-correlation functionals with respect to the prediction of important structural features including cell parameters and the coordination environment of the metal ions. Next, we investigate the excited state properties using DFT and TD-DFT approaches to characterise the emission behaviour. The combined use of full periodic and cluster model approaches at appropriate levels of theory allows one to form a detailed picture of the excited state processes at play in these materials.

\section{Computational Details}

Calculations were performed both on periodic and cluster models for each MOF structure. The computational setup used in each case is described below.

All calculations were carried out using the Crystal14 package ${ }^{23}$ which uses atom-centred Gaussian basis sets. In order to strike a good balance between time and accuracy, the Pople double zeta basis set 6-31G(d) was used in all calculations for all but transition-metal atoms. Zinc and cadmium were described by the basis sets proposed by Pettinger ${ }^{24}$ and $\mathrm{Dou}^{25}$, respectively. The number of k-points in the irreducible Brillouin zone was set to 8 via a shrinking factor of 2 . A total of five exchangecorrelation functionals were tested. This test set was composed of two GGA functionals $\left(\mathrm{PBE}^{26}\right.$ and $\mathrm{BLYP}^{27,28}$ ), two hybrids $\left(\mathrm{PBEO}^{29}\right.$ and $\mathrm{B}^{2} \mathrm{LYP}^{30}$ ) and a functional purposely optimised to yield accurate equilibrium structure properties in solids (bond lengths and lattice parameters) known as $\mathrm{PBEsol}^{31}$.

Structural optimisation of the MOFs and ligand molecular crystal $\left(\mathrm{H}_{2} \mathrm{DMPMB}\right)$ were performed. The Grimme "D2" dispersion correction scheme ${ }^{32}$ was tested within the molecular crystal to better assess 
the extent of interligand interaction that arises from both $\pi-\pi$ stacking and hydrogen bonding interactions.

In order to qualitatively evaluate the emission energy from the triplet state, periodic calculations were also conducted using the SPINLOCK feature of Crystal14, allowing one to fix the difference between the number of alpha and beta electrons. This difference was set to 8 to simulate a triplet excited state centred on the 4 ligands in the unit cell. After the optimisation of this excited state, a single point calculation was conducted for the singlet state while retaining the geometry of the triplet electron configuration in order to quantify the vertical energy gap between the singlet and triplet potential energy surfaces at this point.

All calculations on molecular clusters were conducted using Gaussian $09^{33}$. Calculations were performed on a cluster cut from the periodic structure of each of the M[DMPMB] $(\mathrm{M}=\mathrm{Zn}, \mathrm{Cd})$ systems previously optimised at PBE0 level of theory. Each cluster consists of two metal ions bridged by two DMPMB ligands, with an additional 4 ligands used to complete the metal coordination sphere and terminate the cluster. Hydrogen atoms were added to the uncoordinated pyrazole groups, as shown in Figure 2. TD-DFT calculations were performed in order to calculate the vertical excitation energies and associated oscillator strengths of these clusters. All calculations were again performed with the 6-31G(d) Pople double zeta basis set for all non-metal atoms while the Los Alamos (LANL2) effective core pseudopotential and corresponding double zeta valence basis set were used to describe the cadmium and zinc atoms. The same technique as described above for periodic calculations, while holding the uncoordinated (i.e. extended) nitrogen atoms fixed, was also used to assess the singlettriplet energy gap and, in turn, validate the cluster model used to describe the solid state framework. Additionally, the $\mathrm{S}_{1} \rightarrow \mathrm{S}_{0}$ emission energies of the $\mathrm{H}_{2}$ DMPMB ligand were calculated by optimising its first singlet excited state geometry at TDDFT level for both the free ligand and with the nitrogen atoms constrained to the positions of the calculated MOF structures.

Absorption spectra have been presented as a combination of convoluted Gaussian functions with full width at half maximum height set to $0.3 \mathrm{eV}$ centred at each excitation energy. Computed oscillator strengths are reported in the same figure as vertical lines. 


\section{Results and Discussion}

We first performed full energy-minimization of the $\mathrm{Zn}(\mathrm{DMPMB})$ and $\mathrm{Cd}(\mathrm{DMPMB})$ periodic structures, including both atomic coordinates and unit cell parameters, with our test set of functionals. Figure 3 shows the absolute error obtained for the $a, b$, and $c$ lattice parameters at the ground state for both $\mathrm{Zn}(\mathrm{DMPMB})$ (in blue) and $\mathrm{Cd}(\mathrm{DMPMB})$ (in green). While it is clear that, from the small absolute error relative to the experimental data, all functionals tested perform well in the description of the lattice geometry, the solid-state-tuned PBEsol functional performs best in the prediction of each lattice vector, followed by the PBE0 functional, for both materials.

Figure 4 shows the percentage error in the calculated metal coordination environment relative to the experimental data, again using the functional test set mentioned above, at the ground state. The ability of a given functional to characterise the first coordination sphere of the metal centres was evaluated using two criteria: the error in i) nitrogen-metal-nitrogen coordination angles and in ii) the bond distance between the metal centres and coordinating nitrogen atoms. As with the lattice parameters, the data show that all functionals perform within a low margin of error for all parameters tested across both structures (maximum of $4.0 \%\left(4.4^{\circ}\right)$ for BLYP GGA functional when calculating coordination angles). Surprisingly, given its remit, the PBEsol functional appears to give no appreciable increase in accuracy when calculating either bond lengths or angles and all functionals deliver a similar performance with no single level of theory standing out, in this case, as superior. Furthermore, there is no discernible difference in performance between hybrid and GGA functionals for the calculation of these structural features. From this it can be concluded that, for these MOF systems, the metal coordination environment is not very sensitive to the functional chosen given that GGAs, hybrids and a solids-optimised functional all performed similarly and were able to predict the coordination geometry of the metal sites with sufficient accuracy. Generally, hybrid functionals (such as PBE0 or B3LYP) slightly outperform GGAs approach. The lack of sensitivity of the coordination environment to the choice of functional and general good performance of all those tested rendered this an unimportant factor in the choice of functional and level of theory to be used in excited state calculations. For this reason, PBE0 was chosen for excited state calculations since it gave results of a 
similar quality to the PBEsol functional for structural parameters and as it is known to perform accurately within TD-DFT calculations ${ }^{34,35}$. To test if charge transfer would play a significant role, vertical absorption energies for the $\mathrm{Zn}$ MOF were also calculated using the long-range corrected CAM-B3LYP functional, which is known to correctly treat both valence and charge transfer excitations $^{36,37}$. Results shown in Table S2 show no qualitative difference with respect to those calculated with PBE0 and we will thus only present and discuss the results obtained with PBE0 here. Given the importance of inter-ligand interactions in $\mathrm{MOF}^{38}$, significant dispersion interactions effects can be important in the prediction and characterisation of luminescence phenomena ${ }^{39}$.

In light of this, the PBE0 functional was taken forward in order to investigate the effects of interligand interaction of $\mathrm{H}_{2} \mathrm{DMPMB}$, via the incorporation of dispersion interactions using an empirical pairwise potential as proposed by Grimme ${ }^{32}$ (the corresponding results being labelled as PBE0Dispersion). The results are summarised in Table 1 . The $\mathrm{H}_{2}$ DMPMB molecular crystal was chosen as a relevant test case as there are two potential inter-ligand interaction types within its structure: i) intermolecular hydrogen bonds between the hydrogen and nitrogen atoms located on the pyrazole moieties and ii) $\pi-\pi$ stacking between the biphenyl parts. Table 1 shows that the inclusion of the dispersion interactions roughly halves both the error in the hydrogen bond interaction, here monitored by the pyrazole nitrogen to nitrogen intermolecular distance ( $1.35 \%$ to $0.55 \%)$, and the $\pi-\pi$ stacking distance $(5.06 \%$ to $2.88 \%)$, with a small increase in error with respect to the primitive cell volume $(0.38 \%$ to $0.71 \%)$ and density $(0.33 \%$ to $0.64 \%)$. This slight loss of accuracy with respect to the cell volume and density can be attributed to the tendency of the Grimme correction scheme to overestimate the long-range electron correlations that are responsible for intermolecular van der Waals forces. At first, it seems strange that the stacking distance is underestimated by the PBE0 functional alone (without dispersion correction) - suggesting that the PBE0 functional itself overestimates these interactions - but this is an indirect effect, due to the hydrogen bonding between ligands being poorly represented. Indeed, looking at the two-dimensional, layered structure of the molecular crystal Figure 5, weaker hydrogen bonding interactions allow the coordinating pyrazole groups to spread further apart and, in turn, reduce the distance between biphenyl moieties. The 
introduction of the dispersion interactions better describes both the hydrogen bonding and $\pi-\pi$ stacking, pulling the ligands closer together at the pyrazole parts and further apart with respect to the biphenyl groups. This effect is rooted in the two-dimensional nature of the molecular crystal and is not a result of the PBE0 functional somehow overestimating the inter-ligand stacking interactions between biphenyl groups with respect to the addition of dispersion interactions.

Figure 6 shows the calculated vertical absorption spectra previously described using cluster models of the cadmium and zinc MOFs (as shown in Figure 2) along with calculated absorption and emission spectra of the free ligand. The calculated absorption spectrum for the free ligand shows one, single band located at $259 \mathrm{~nm}$, as shown in Figure 2. This band corresponds to a $\pi \rightarrow \pi^{*}$ transition centred on the biphenyl moiety. All computed vertical transition energies are explicitly reported in the Supporting Information.

Overall, the spectra of the three compounds are actually dominated by this intense ligand centred (LC) transition which is only slightly tuned by the MOF environment. Importantly, at least for singlet-tosinglet transitions, no other "bright" transitions were computed within the energy range of interest. Here, a "bright" transition is deemed to be a transition with a calculated oscillator strength significantly greater than zero.

As for the free ligand, for the zinc and cadmium MOF clusters an absorption band of $\pi-\pi^{*}$ character corresponding to the biphenyl-centred excitation of the ligand was determined and agrees well with the absorption band calculated for the free ligand itself at $264 \mathrm{~nm}$.

Now we shall consider the emission properties of these materials. Experimentally, the photoluminescence has been ascribed to LC states, which is consistent with the fact that the LC computed transition is the only bright transition dominating the spectra of the compounds. Nonetheless, the origin of the large shift observed in emission going from the $\mathrm{Zn}$ to $\mathrm{Cd}$ based MOF it is not obvious or clear. To clarify this point - following the reasoning which supposes a LC emission the emission energies for the M[DMPMB] clusters and $\mathrm{H}_{2}$ DMPMB ligand were computed and they are reported, along with those derived from experiment ${ }^{22}$, in Table 2 Note that the determination of 
the emission energy from the singlet excited state $\left(\mathrm{S}_{1} \rightarrow \mathrm{S}_{0}\right)$ (calculated via the full optimisation of the $\mathrm{S}_{1}$ excited state geometry) was undertaken for the free ligand only, while the energy of the phosphorescence $\left(\mathrm{T}_{1} \rightarrow \mathrm{S}_{0}\right)$ was determined as described in the computational details section.

The emission wavelength from the singlet $\pi-\pi^{*}$ excited state of the free ligand was calculated at $332 \mathrm{~nm}$. Fixing the nitrogen atom positions constrained in the coordination geometry for both $\mathrm{Zn}[\mathrm{DMPMB}]$ and $\mathrm{Cd}[\mathrm{DMPMB}]$, the same calculations of the emission wavelength from the singlet $\pi-\pi^{*}$ excited state with were undertaken to decouple any perturbative effects of the metal with those of the geometrical constraints imposed on the ligand by the metal within the MOF structures. It was found that this geometrical constraint had little effect as shown by both the calculated absorption and emission values for the ligand with constrained nitrogen on the pyrazole groups corresponding to the optimised solid-state structures for Zn[DMPMB] (4.44 eV -279 nm- absorption; $3.78 \mathrm{eV}$-328 nmemission) and Cd[DMPMB] (4.48 eV -277 nm- absorption; $3.79 \mathrm{eV}-327 \mathrm{~nm}$ - emission). Comparing the calculated emission energy for the free ligand or the ligand constrained at Cd[DMPMB] geometry (332 $\mathrm{nm}$ and $327 \mathrm{~nm}$, respectively) and the experimentally observed emission value for the $\mathrm{Cd}[\mathrm{DMPMB}]$ species $(3.70 \mathrm{eV}, 335 \mathrm{~nm})$, it is clear that the emission from the cadmium based MOF can be ascribed confidently to a ligand centred transition, as previously predicted in the experimental work $^{22}$. This is also shown pictorially in Figure 6. Upon comparing the calculated emission energy from the singlet $\pi-\pi^{*}$ excited state of the free ligand or the ligand constrained at $\mathrm{Zn}$ [DMPMB] geometry (332 nm and $328 \mathrm{~nm}$, respectively) and the experimental emission energy for Zn[DMPMB] $(2.81 \mathrm{eV}, 441 \mathrm{~nm})$ it is clear that the emission shown by the zinc based MOF is not adequately explained by ligand centred emission from the singlet excited state. Furthermore, as seen above, accounting for the geometrical constraints imposed by coordination environment in $\mathrm{Zn}$ [DMPMB] does not account for this shift in emission energy with respect to the free ligand. Lastly, given the experimental excitation energy used in the case of $\mathrm{Zn}[\mathrm{DMPMB}](3.40 \mathrm{eV})$, it is unlikely that the emission observed in this MOF is a result of the same process as that observed for Cd[DMPMB] since the $\pi-\pi^{*}$ ligand centred excited state is predicted to be higher - vertically - than the excitation energy experimentally used (computed at $264 \mathrm{~nm}$ ). 
If the $\mathrm{Zn}[\mathrm{DMPMB}]$ emission does not stem from the ligand-centred singlet $\pi-\pi^{*}$ state, we are left with the option of this luminescence behaviour resulting either from another singlet excited state (fluorescence $\left.\left(\mathrm{S}_{1} \rightarrow \mathrm{S}_{0}\right)\right)$ that can be accessed using the experimental absorption energy of $3.40 \mathrm{eV}$ or from the triplet $\pi-\pi^{*}$ excited state (phosphorescence $\left(\mathrm{T}_{1} \rightarrow \mathrm{S}_{0}\right)$ ) centred on the ligand.

As previously discussed for the absorption spectra of the $\mathrm{Zn}$ and $\mathrm{Cd}$ MOF clusters, there are no other singlet excited states with appreciable oscillator strength that lie within the required energy region and therefore are not likely to be at the origin of the intense photoluminescence properties observed for the $\mathrm{Zn} \mathrm{MOF.} \mathrm{Therefore} \mathrm{an} \mathrm{emission} \mathrm{from} \mathrm{the} \mathrm{triplet,} \mathrm{which} \mathrm{has} \mathrm{been} \mathrm{observed} \mathrm{in} \mathrm{other} \mathrm{d}^{10}$ metalorganic complexes ${ }^{20,21}$ could provide a reasonable explanation for the difference in fluorescence behaviour between the zinc and cadmium based MOFs. Using the cluster approach, the triplet excited states were vertically computed from the ground state $\mathrm{Zn}$ and Cd MOF geometries at 378 and $371 \mathrm{~nm}$, respectively. Therefore upon irradiation at $365 \mathrm{~nm}$, as in experiment, this state can be populated. Indeed, although this type of transition is spin forbidden, the presence of a metal ions allows their efficient population through spin-orbit coupling.

In addition to the emission energies from the singlet $\pi-\pi^{*}$ state, the emission energies from the triplet $\pi-\pi^{*}$ of the free ligand and $\mathrm{Zn}$ and Cd MOFs were also estimated using the previously described methodology. From an electronic density-difference plot $\left(\rho_{\alpha}-\rho_{\beta}\right)$ (Figure S1), it can be seen that this triplet excited state is indeed centred on the biphenyl moiety of the ligand. The emission from the triplet excited state of the free ligand was found to be significantly red shifted (with respect to the singlet emission energy) at $554 \mathrm{~nm}(2.24 \mathrm{eV})$. The emission energies estimated from the triplet excited states of the constrained ligand geometries were found to be slightly blue shifted with respect to the free ligand $(522 \mathrm{~nm} ; 2.38 \mathrm{eV})$ for both $\mathrm{Cd}[\mathrm{DMPMB}]$ and $\mathrm{Zn}[\mathrm{DMPMB}]$. Furthermore, phosphorescence wavelengths from the $\pi-\pi^{*}$ state were calculated using both cluster (Figure 2) and periodic models. Beginning with the cluster model for $\mathrm{Zn}[\mathrm{DMPMB}]$, a blue shift of $0.27 \mathrm{eV}$ is observed relative to the free ligand phosphorescence from the $\pi-\pi^{*}$ state while a slightly smaller blue shift of $0.17 \mathrm{eV}$ is determined via periodic calculations. For Cd[DMPMB], a smaller blue shift of 
$0.01 \mathrm{eV}$ is determined using the cluster model. From this, the effect of the environment is clearly observed on the emission energy from the triplet $\pi-\pi^{*}$ excited state.

Considering the emission energy observed in experiment and the calculated energy difference between the $T_{1}$ and $S_{0}$ surfaces for the $\mathrm{Zn}[\mathrm{DMPMB}]$ cluster at the optimised $\mathrm{T}_{1}$ geometry, the results are comparable (2.50 eV calculated, $2.81 \mathrm{eV}$ in experiment). This leads to the conclusion that the emission observed for the zinc compound could, indeed, also be a result of phosphorescence from a $\pi-\pi^{*}$ triplet excited state. It is also worth noting that, according to calculations conducted using PBE0, there is no appreciable change in coordination geometry between the optimised singlet ground state and triplet state of either MOF.

Finally, the small discrepancy between the $\mathrm{T}_{1} \rightarrow \mathrm{S}_{0}$ energy values calculated using the cluster model and periodic calculations $(0.09 \mathrm{eV}$ for $\mathrm{Zn}[\mathrm{DMPMB}] ; 0.059 \mathrm{eV}$ for $\mathrm{Cd}[\mathrm{DMPMB}])$ allows one to conclude that the cluster model used to determine absorption energies via TDDFT calculations is sufficiently accurate. This also illustrates that these excitations are local processes in MOFs and could validate further use of similar cluster models to study excited state processes in metal-organic frameworks.

Overall, this result is surprising as it is indeed counterintuitive that the MOF structure based on the lighter of the two transition metals would promote intersystem crossing and provide triplet emission. Though it can't be completely ruled out that a higher-lying triplet state may play a role in the case of Cd[DMPMB], based on the present calculations it seems plausible that the large shift observed going from $\mathrm{Zn}$ to $\mathrm{Cd}$ is related to the different nature of the emissive state for the two compounds.

\section{Conclusions}

Rational design of useful optical properties within metal-organic frameworks is a subject of ever increasing attention in recent years with theoretical approaches poised to play a key role alongside experiment in the understanding further development of high performance materials. We have demonstrated a computational protocol rooted in quantum mechanical calculations at DFT and TDDFT level able to successfully discriminate between subtle luminescence mechanisms in a pair of 
isostructural MOFs with interchangeable metal cations. This pair of systems is a novel example of tuneable luminescence in MOFs with an approximately $0.88 \mathrm{eV}$ (441 nm to $335 \mathrm{~nm}$ ) blue shift going from the zinc based to cadmium based structure. This method was able to confirm the higher energy emission seen from the cadmium-based structure is a result of a ligand-centred emission from the singlet $\pi-\pi^{*}$ excited state. The luminescence behaviour resulting from the zinc-based structure was found to be more complex, with luminescence possibly resulting from the triplet $\pi-\pi^{*}$ excited state (localised on the ligand).

The effectiveness of this protocol in the discrimination of excited state processes in MOFs at relatively low computational cost can be a valuable tool in the design and understanding of high performance optical materials.

Generally, the effectiveness of this protocol in its discrimination of excited state processes, ability to cover luminescence types from both singlet and triplet states and general applicability in recovering both geometrical and photophysical features of MOFs at relatively low computational cost can be a valuable tool in the design and understanding of high performance optical materials. 


\section{References}

1. H. C. Zhou, S. Kitagawa, Chem. Soc. Rev., 2014, 43, 5415-5418

2. J. Wang, L. Huang, R. Yang, Energy \& Environmental Science, 2014, 7, 3478-3518

3. A. Dhakshinamoorthy, A. M. Asiri, H. Garcia, Chem. Comm., 2014, 50, 12800-12814

4. Z. Hu, B. J. Deibert, J. Li, Chem. Soc Rev. 2014, 43, 5815

5. S. O. Odoh, C. J. Cramer, D. G. Truhlar, L. Gagliardi, Chem. Rev. 2015, 115, 6051-6111

6. F-X. Coudert, A. H. Fuchs, Coord. Chem. Rev. 2016, 307, 211-236

7. L. Sarkisov, J. Kim, Chemical Engineering Science, 2015, 121, 332-330

8. J. Heine, K. Müller-Buschbaum, Chem. Soc. Rev., 2013, 42, 9232

9. C. L. Jones, A. J. Tansella, T. L. J. Easun, Mater. Chem. A, 2016, 4, 6714-6723

10. F-X. Coudert, Chem. Mater., 2015, 27, 1905-1916]

11. M. D. Allendorf, C. A. Bauer, R. K. Bhakta, R. T. J. Houk, Chem. Soc. Rev., 2009, 38, 13301352

12. Y. Cui, Y. Yue, G. Qian, B. Chen, Chem. Rev. 2012, 112, 1126-1162

13. D. F. Sava, L. E. S. Rohwer, M. A. Rodriguez, T. M. Nenoff, J. Am. Chem. Soc., 2012, 134, 3983-3986.

14. Y. Hasegawa, T. Nakanishi, $R S C A d v .2015, \mathbf{5}, 338-353$

15. S. A. Sapchenko, D. G. Samsonenko, V. P. Fedin, Polyhedron, 2013, 55, 179-183

16. C. L. Whittington, L. Wojtas, R. W. Larsen, Inorg. Chem., 2014, 53, 160-166

17. M. Ji, X. Lan, Z. Han, C. Hao, J. Qui, Inorg. Chem., 2012, 51, 12389-12394

18. Y. Yue, A. J. Binder, R. Song, Y. Cui, J. Chen, D. K. Hensley, S. Dai, Dalton Trans., 2014, 43, 17893

19. D. Yan, Y. Tang, H. Lin, D. Wang, Scientific Reports, 2014, 4, 4437

20. W. Y. Wong, L. Liu, J. X. Shi, Angew. Chem. Int. Ed. 2003, 42, $4064-4068$

21. H-Y. Chao, W. Lu, Y. Li, M. Chan, C-M. Che, K-K. Cheung, N. Zhu, J. Am. Chem. Soc., 2002, 124, 14696-14706

22. A. Tabacaru, C. Pettinari, F. Marchetti, S. Galli, N. Masciocchi, Cryst. Growth Des. 2014, 14, $3142-3152$

23. R. Dovesi, R. Orlando, B. Civalleri, C. Roetti, V. R. Saunders, C. M. Zicowich-Wilson, Krist., 2009, 220, 571-573

24. M. F. Pettinger, O. Vilela, T. J. Bredow, Comput. Chem., 2013, 34, 451-459

25. Y. Dou, R. G. Egdell, D. S. L. Law, N. M. Harrison, B. G. J. Searle, Phys. Cond. Matter, 1998, 10, 8447-8458

26. J. P. Perdew, K. Burke, M. Ernzerhof, Phys. Rev. Lett., 1996, 77, 3865.

27. A. D. Becke, Phys. Rev. A, 1998, 38, 3098

28. C. Lee, W. Yang, R. G. Parr, Phys. Rev. B, 1988, 37, 785.

29. C. Adamo, V. Barone, J. Chem. Phys., 1999, 110, 6158-6170.

30. A. D. Becke, J. Chem. Phys., 1993, 98, 5648-5652.

31. J. P. Perdew, A. Ruzsinszky, G. I. Csonka, O. A. Vydrov, G. E. Scuseria, L. A. Constantin, X. Zhou, K. Burke, Phys. Rev. Lett., 2008, 100, 136406

32. S. Grimme, J. Comput. Chem., 2006, 27, 1787-1799

33. M. J. Frisch, G. W. Trucks, H. B. Schlegel, G. E. Scuseria, M. A. Robb, J. R. Cheeseman, G. Scalmani, V. Barone, B. Mennucci, G. A. Petersson, H. Nakatsuji, M. Caricato, X. Li, H. P. Hratchian, A. F. Izmaylov, J. Bloino, G. Zheng, J. L. Sonnenberg, M. Had, and D. J. F. Gaussian 09, Revision D.01. Gaussian Inc., Wallingford CT. 2009.

34. C. Adamo, G. E. Scuseria, V. Barone, J. Chem. Phys., 1999, 111, 2889

35. D. Jaquemin, V. Wathelet, E. A. Perpète, C. Adamo, J. Chem. Theo. Comput., 2009, 5, 24202435

36. D. Jacquemin, E. A. Perpète, G. E. Scuseria, I. Ciofini, C. Adamo, J. Chem. Theo. Comput., $2008,4,123-135$

37. A. Dreuw, J. L. Weisman, M. Head-Gordon, J. Chem. Phys., 2003, 119, 2943

38. Q. Fang, G. Zhu, X. Shi, G. Wu, G. Tian, R. Wang, S. Qui, Journal of Solid State Chemistry, 2004, 117, 1060-1066 
39. D. Presti, F. Labat, A. Pedone, M. J. Frisch, H. P. Hratchian, I. Ciofini, M. C. Meziani, C. Adamo, J. Chem. Theory Comput., 2014, 10, 5577-5585 
Table 1. Calculated and experimental values of key structural parameters investigated on the $\mathrm{H}_{2}$ DMPMB ligand molecular crystal. Nitrogen to Nitrogen Intermolecular distance is defined as that between closest neighbouring nitrogen atoms in the crystal and taken as a measure of intermolecular hydrogen bond features.

\begin{tabular}{cccc} 
Functional & PBE0 & PBE0 + Dispersion & Experimental $^{22}$ \\
\hline Nitrogen-Nitrogen distance $(\AA)$ & 2.935 & 2.912 & 2.896 \\
Unit Cell Volume $\left(\AA^{3}\right)$ & 1017.3 & 1006.2 & 1013.4 \\
Density $\left(\mathrm{g} / \mathrm{cm}^{3}\right)$ & 1.209 & 1.222 & 1.213 \\
$\boldsymbol{\pi}-\boldsymbol{\pi}$ Stacking Distance $(\AA)$ & 3.658 & 3.742 & 3.853
\end{tabular}


Table 2. Calculated main absorption and emission energies for $\mathrm{H}_{2} \mathrm{DMPMB}, \mathrm{Zn}$ [DMPMB] and $\mathrm{Cd}[\mathrm{DMPMB}]$ along with experimental data. Calculations were also conducted holding the position of the nitrogen atoms of the ligand constrained to that computed at the ground state at PBE0 level for $\mathrm{Zn}[\mathrm{DMPMB}]$ and $\mathrm{Cd}[\mathrm{DMPMB}]$ labelled constrained $\mathrm{N}_{\mathrm{Zn}}$ and constrained $\mathrm{N}_{\mathrm{Cd}}$ respectively.

\begin{tabular}{|c|c|c|c|c|}
\hline \multirow[b]{2}{*}{ Model } & \multicolumn{3}{|c|}{ Calculated } & \multirow{2}{*}{$\begin{array}{c}\text { Experimental }^{22} \\
\lambda_{\mathrm{em}}(\mathrm{nm})\end{array}$} \\
\hline & $\lambda_{\mathrm{abs}}(\mathrm{nm})$ & $\begin{array}{l}\lambda_{\mathrm{em}}(\mathrm{nm}) \\
\left(\mathrm{S}_{1} \rightarrow \mathrm{S}_{0}\right)\end{array}$ & $\begin{array}{c}\lambda_{\mathrm{em}}(\mathrm{nm}) \\
\left(\mathrm{T}_{1} \rightarrow \mathrm{S}_{0}\right)\end{array}$ & \\
\hline $\begin{array}{c}\mathrm{H}_{2} \mathrm{DMPMB} \\
\text { (Free) }\end{array}$ & 285 & 332 & 554 & - \\
\hline $\begin{array}{c}\mathrm{H}_{2} \mathrm{DMPMB} \\
\text { (Constrained } \\
\left.\mathrm{N}_{\mathrm{Zn}}\right)\end{array}$ & 279 & 328 & 522 & - \\
\hline $\begin{array}{c}\mathrm{H}_{2} \mathrm{DMPMB} \\
\text { (Constrained } \\
\left.\mathrm{N}_{\mathrm{Cd}}\right)\end{array}$ & 277 & 327 & 522 & - \\
\hline $\begin{array}{c}\text { Cd[DMPMB] } \\
\text { (Cluster) }\end{array}$ & 269 & nc & 520 & \\
\hline $\begin{array}{c}\mathrm{Cd}[\mathrm{DMPMB}] \\
\text { (Periodic) }\end{array}$ & $\mathrm{nc}$ & $\mathrm{nc}$ & 515 & \\
\hline $\begin{array}{c}\mathrm{Zn}[\mathrm{DMPMB}] \\
\text { (Cluster) }\end{array}$ & 266 & nc & 495 & $41^{\mathrm{b}}$ \\
\hline $\begin{array}{c}\mathrm{Zn}[\mathrm{DMPMB}] \\
\text { (Periodic) }\end{array}$ & nc & nc & 514 & \\
\hline
\end{tabular}

$n c=$ not computed 


\section{Captions to Figures}

Figure 1. (a) View along cell vector $\mathrm{c}$ showing unit cell; (b) protonated $\mathrm{H}_{2} \mathrm{DMPMB}$ ligand structure; (c) view along cell vector $\mathrm{b}$ showing metal coordination environment and infinite network

Figure 2. Cluster model taken along the $1 \mathrm{D}$ inorganic chain within M[DMPMB] used for TDDFT calculations showing metal cation (red), coordinating nitrogen atoms (blue) and organic linker with carbon (black) and hydrogen (white).

Figure 3 Histogram showing absolute error (in $\AA$ ) in calculated lattice vectors relative to experimental data. Results obtained for $\mathrm{Zn}(\mathrm{DMPMB})$ and $\mathrm{Cd}(\mathrm{DMPMB})$ are represented as blue and green bars, respectively

Figure 4. Histogram showing average percentage error in calculated coordination angles and coordination bond distances relative to experimental data. Results obtained for $\mathrm{Zn}(\mathrm{DMPMB})$ and $\mathrm{Cd}(\mathrm{DMPMB})$ are represented as blue and green bars, respectively.

Figure 5. Structural view of 2 adjacent unit cells of $\mathrm{H}_{2} \mathrm{DMPMB}$ ligand molecular crystal. Hydrogens are omitted for clarity. Green arrow indicates hydrogen bond distance defined via intermolecular nitrogen distance.

Figure 6. Calculated absorption spectra for clusters of $\mathrm{Zn}[\mathrm{DMPMB}]$ and $\mathrm{Cd}[\mathrm{DMPMB}]$ along with simulated absorption and emission spectra for the free $\mathrm{H}_{2} \mathrm{DMPMB}$ ligand. The wavelength of the experimentally observed emission maxima for both MOF species are also indicated. 


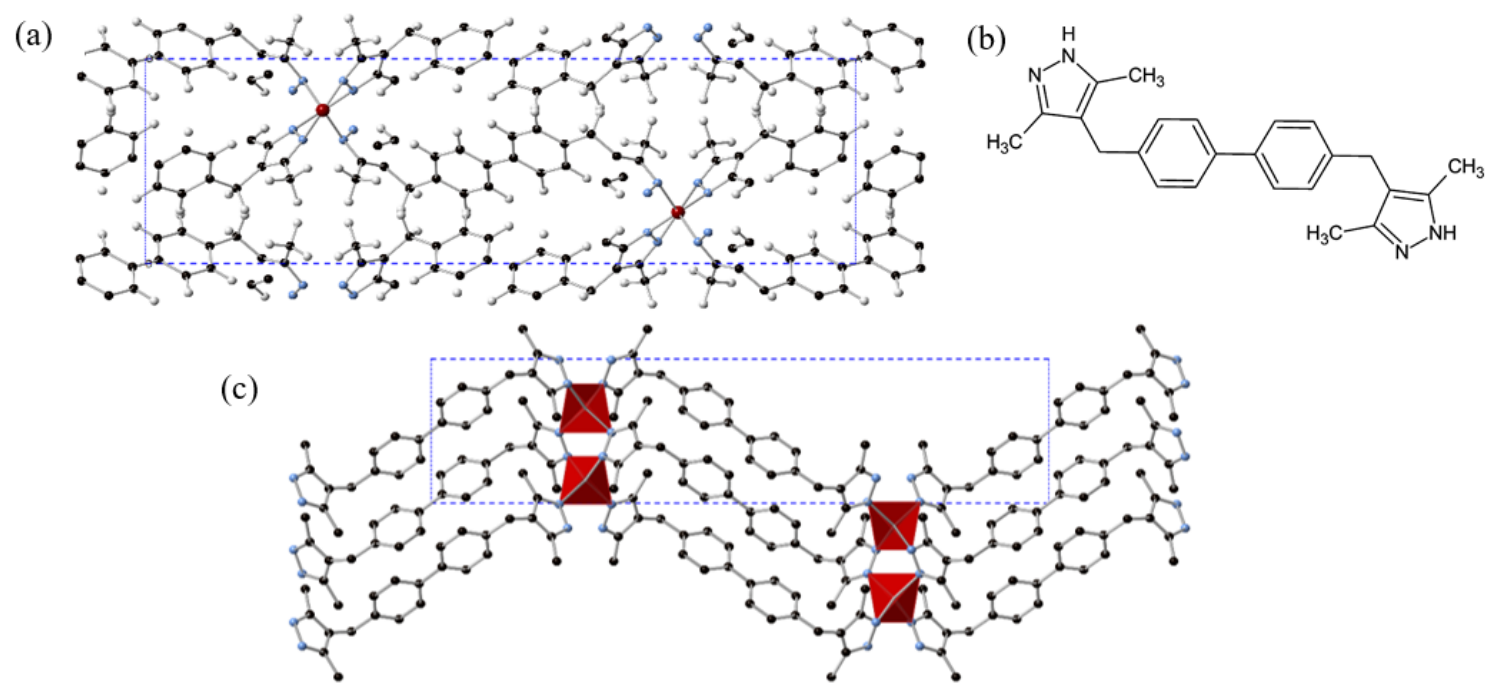

Figure 1 


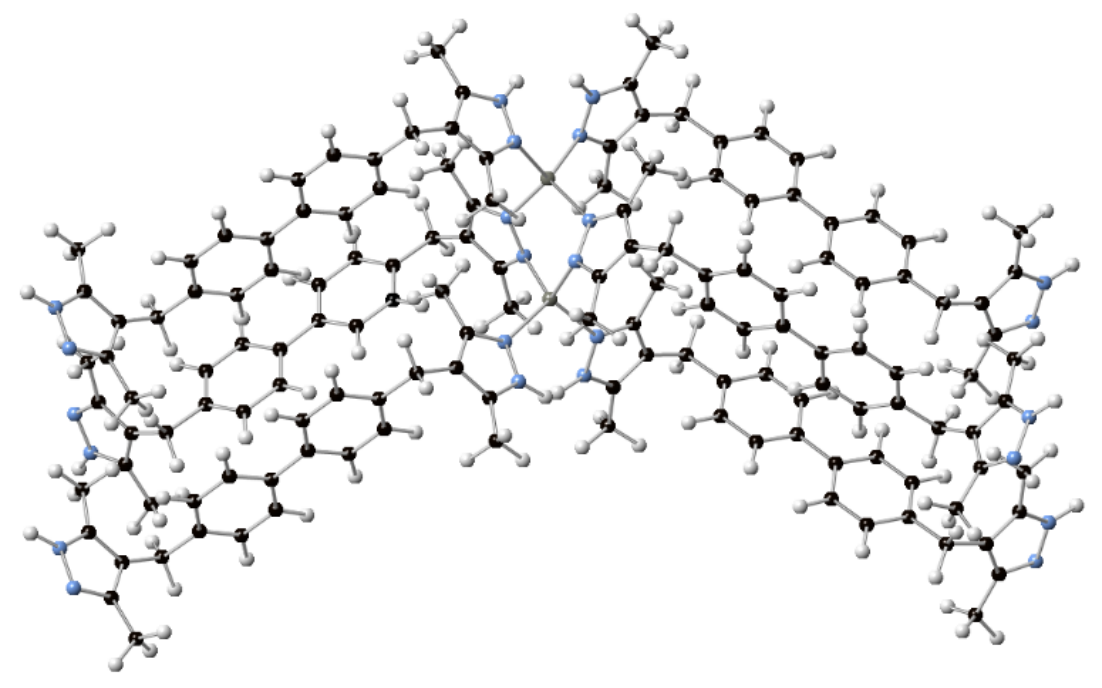

Figure 2 


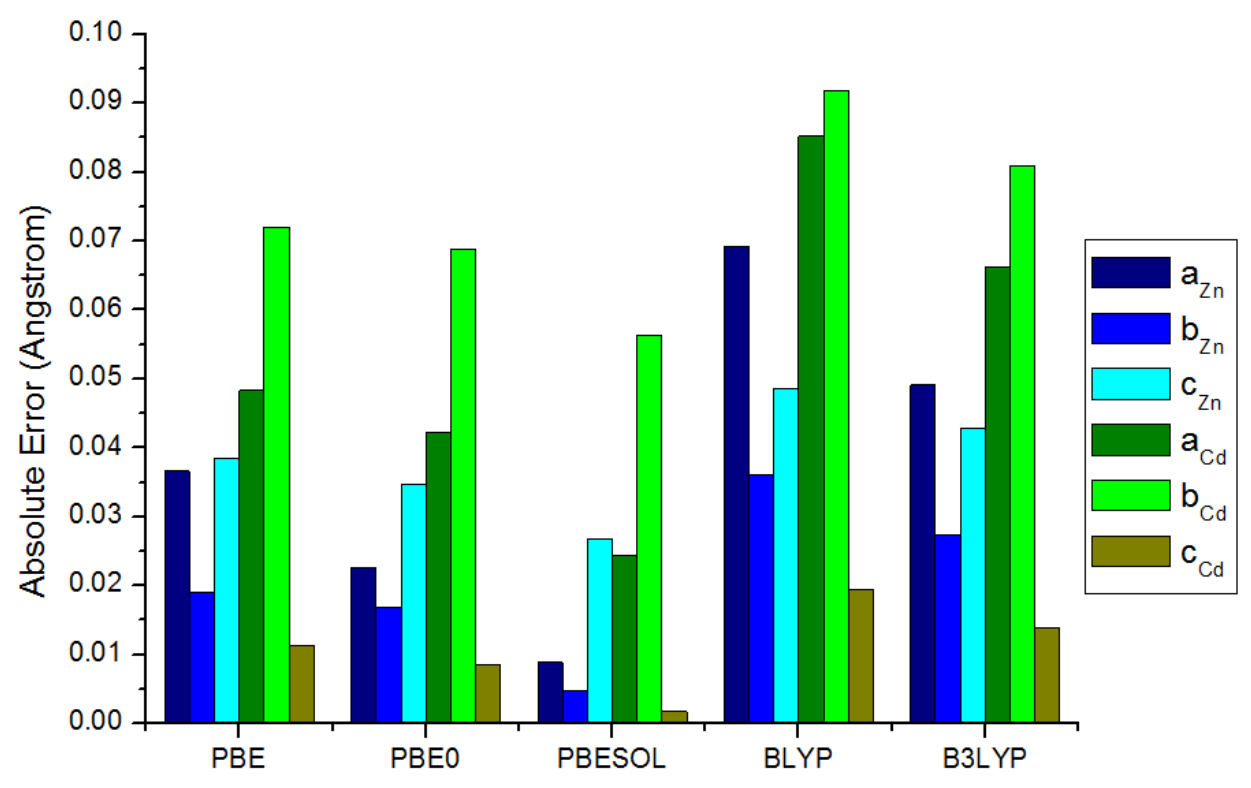

Figure 3 


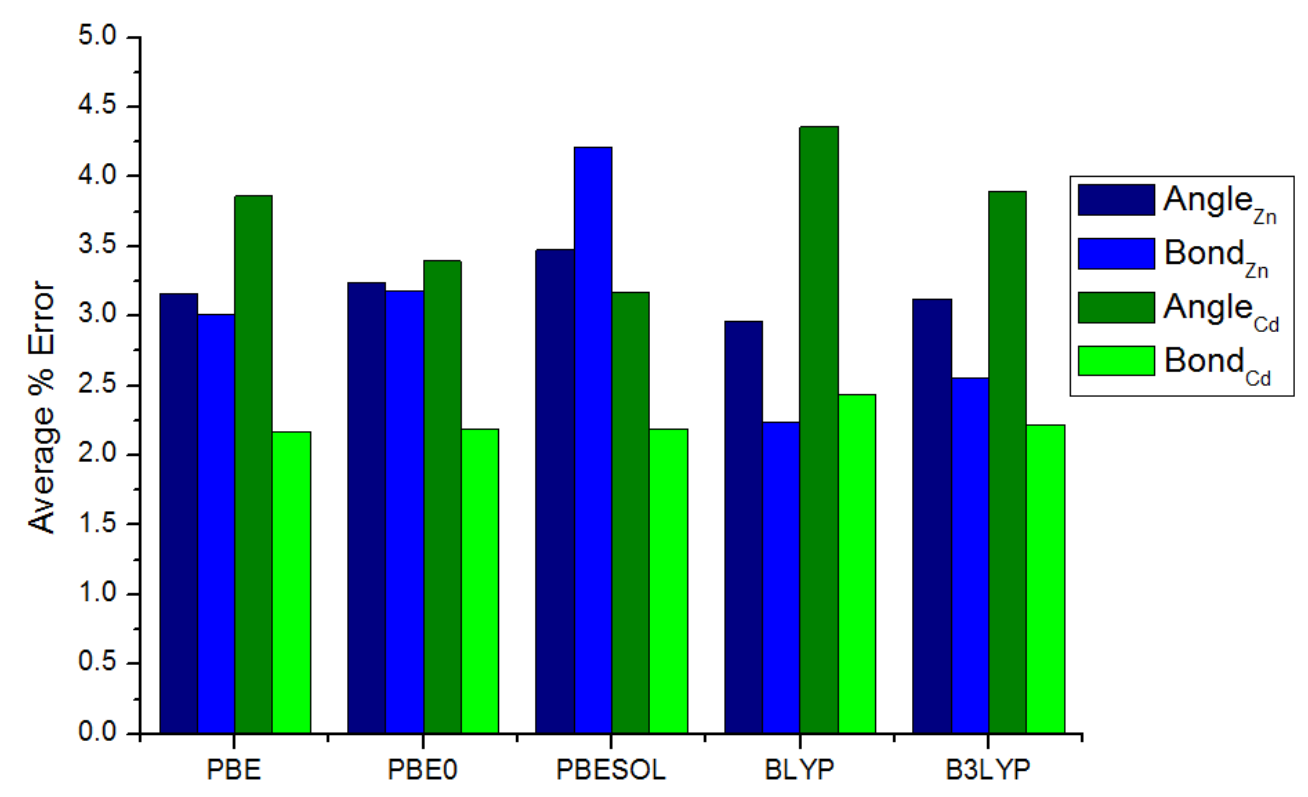

Figure 4 


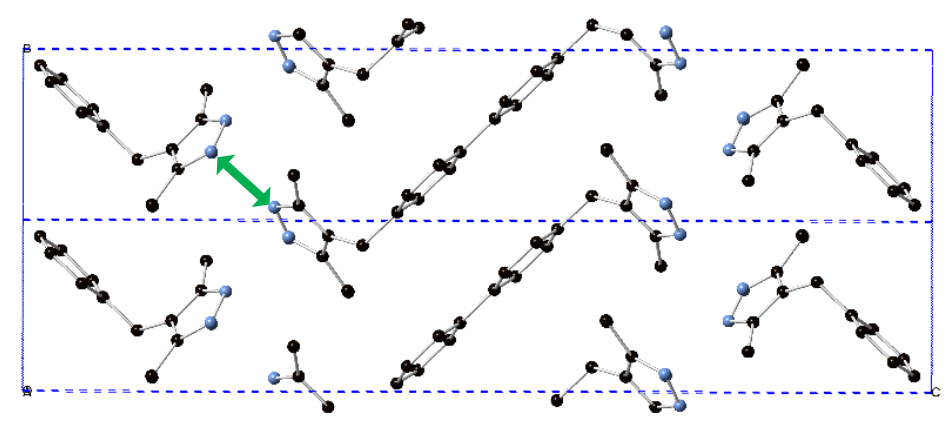

Figure 5 


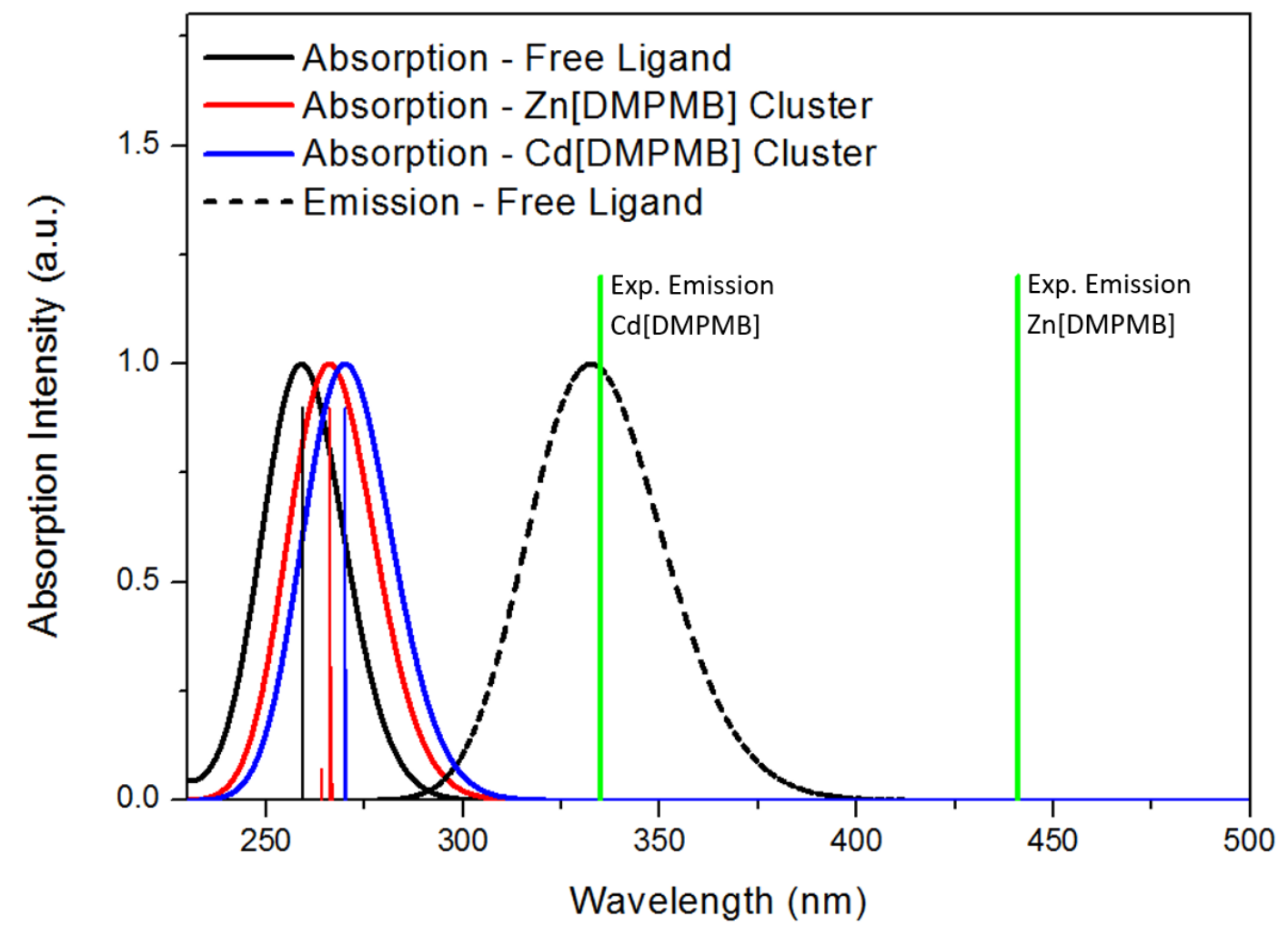

Figure 6 